\title{
Effect of Voltage Reduction in Minimising Partial Discharge Activity in Cables - Experimental Study
}

\author{
Faisal Peer Mohamed, W H Siew, Bojie Sheng, Brian Stewart \\ Electrical Plant Diagnostics Research Group, University of Strathclyde, Glasgow, UK
}

\begin{abstract}
A common cause of insulation degradation in high voltage (HV) cables is due to partial discharges (PD). PD may be initiated through imperfections and contaminants present in the insulation. It can be hypothesized that a reduction in system voltage can potentially reduce $P D$, which will correspondingly extend the service life of the cable. Currently, industry voltage statutory requirements permit $\pm 6 \%$ tolerance setting on nominal voltage on distribution networks. Such $\pm 6 \%$ voltage reduction on may have little or adverse effect on PD magnitude which depends on the nature of defect. Hence the accuracy and type of measuring PD while reducing system voltage is more critical for this application. Power cable as a low pass filter which attenuates and disperse the PD pulses. In this paper effect of voltage reduction on $P D$ is investigated using standard electrode geometries such as point-plane, point-point and point- rod using IEC60270 measurement system together with frequency domain measurements. It has been observed that PD frequency characteristics at different voltages varies in the wideband frequency spectrum.
\end{abstract}

\section{INTRODUCTION}

In the UK, most of the cables installed in the 1950s and 1960s are approaching the end of their designed life, according to manufacturer guidelines. This is likely to lead to a prevalence of circuit faults resulting directly from insulation degradation and breakdown because of ageing[1]. A program for the wholesale replacement of these vast cable networks is impracticable and economically unviable[2]. Hence there is a clear requirement to control or minimize PD activity. The term "Partial Discharge (PD)" is defined by IEC 60270 (Partial Discharge Measurements) as "a localized electrical discharge that partially bridges the insulation between conductors and which may or may not occur adjacent to a conductor" $[2,3]$. Based on the voltage dependence property of $\mathrm{PD}$, it can be hypothesized that a reduction in system voltage can potentially reduce $\mathrm{PD}$, which may correspondingly extend the service life of the cable. Currently, Standards Regulations permit $\pm 6 \%$ tolerance on the nominal voltage of low voltage networks. The $\pm 6 \%$ voltage variation may have significant effect on the PD magnitude.

Due to PD activity, pulses having fast rise times are induced across the high voltage and ground electrode. Conventionally, these pulses are captured in the time domain using high speed digitizers from the output of the high frequency CT clamped around the ground conductor. The emitted PD pulses have a wide-band spectrum and they propagate to both ends of a cable while undergoing attenuation and dispersion due to the high frequency propagation characteristics of the cable. Energy contained in the PD pulses is distributed across the various frequency components within the wideband spectrum. The aforementioned $\pm 6 \%$ voltage variation may have a significant effect on PD magnitude which in turn depends on the type of defect[4]. In this paper, in addition to data based on IEC 60270 measurements, PD pulses are also measured using spectrum analyzer (PDS200) having a fixed resolution bandwidth of $120 \mathrm{kHz}$. In addition to that, PD measurements were also conducted with a narrow band filter configuration to study the distribution of PD pulses; namely number of PDs and average PD magnitude across various frequencies within the wide-band frequency spectrum. The reason for making measurements using a selection of standard gap profiles is to have a bench mark on the effect of voltage variation on PD emission. When measurements are then made on-site, it is hoped that the bench marking would serve as a guide on the type of PDs being observed. Experimental setup and test results are discussed in the next section.

\section{EXPERIMENTAL SETUP}

All the electrodes used are made of steel to avoid erosion due to the discharges. Dimensions of electrodes used in various electrode configurations are tabulated as shown in Table 1.

\begin{tabular}{|c|c|l|}
\hline S1 No & Type of Electrode & Dimensions \\
\hline 1 & Pin & Tip diameter: $0.4 \mathrm{~mm}$ \\
\hline 2 & Plane & Height: $10 \mathrm{~mm}$, diameter, $19 \mathrm{~mm}$ \\
\hline 3 & Rod & Height: $27.5 \mathrm{~mm}$, radius of curvature, $2.5 \mathrm{~mm}$ \\
\hline
\end{tabular}

Table 1 Electrode specification

Test setup used in frequency domain measurement of PD is shown in Figure 1 which is basically an IEC 60270 measurement system. In addition to that, a high frequency current transformer (HFCT) having a frequency response of $100 \mathrm{kHz}-20 \mathrm{MHz}$ is clamped around the low voltage electrode. The HFCT output is connected to a spectrum analyzer (PDS200) for scanning of PD activity and also for narrow band PD measurement in time domain.

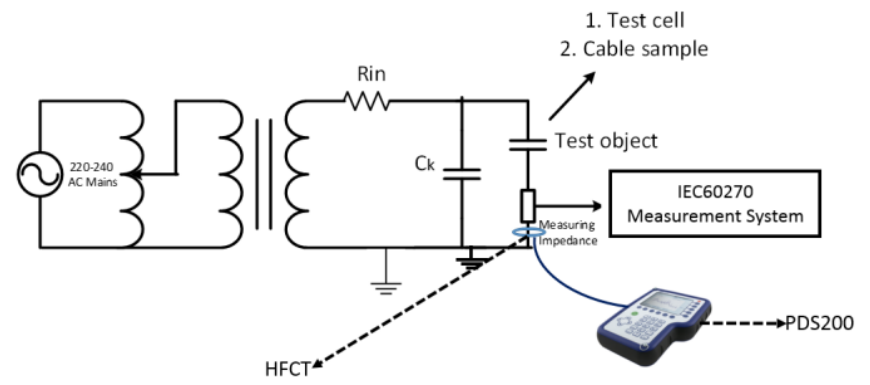

Figure 1 PD measurement setup

Test cell containing electrode configuration connected to the measurement system as shown in Figure 1 is energized until appreciable PD is recorded by IEC60270 measurement system. Spectrum analyzer measures the frequency spectrum after sufficient frequency sweeps. Phase resolved PD pattern 
are derived at various frequencies. Measurements are repeated with voltage reduction of 6 and $12 \%$. Electrode gaps such as point-plane, point-point and point-rod in the test cell as shown in Figure 2 would be used. The test results obtained are discussed in the following sections.

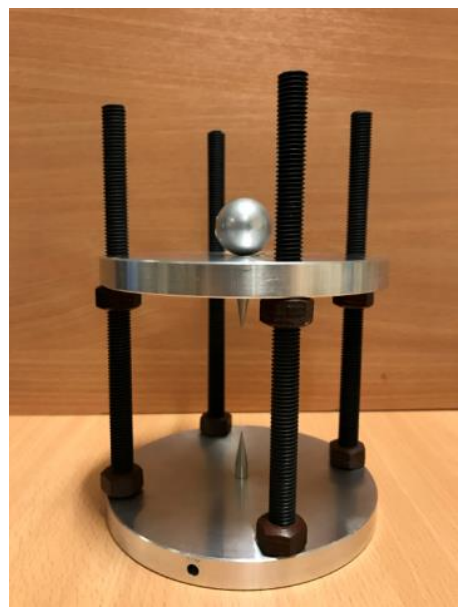

Figure 2 Test cell

III. POINT-PLANE ELECTRODE

Point-plane gap was energized until appreciable PD magnitude appears in both positive and negative cycle of the $50 \mathrm{~Hz}$ applied voltage. Figure 3 shows the $\phi-q-n$ plot of the PD measurement. High peaks of PD magnitude were found in positive half cycle.

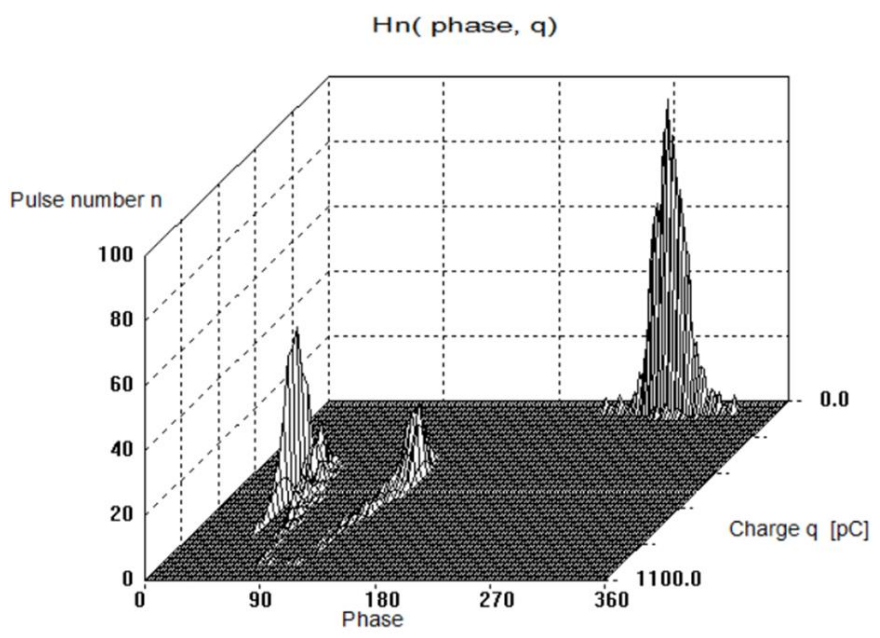

Figure $3 \phi$-q-n Plot (IEC60270)

(Point-Plane, $11.7 \mathrm{kV}$ peak)

Frequency domain measurements of the signals available from the output of HFCT using spectrum analyzer is shown in Figure 4 which has a frequency span from $100 \mathrm{kHz}$ to $50 \mathrm{MHz}$ and which contains all of the signals in the measurement environment including PD from the test cell. From the narrowband PD measurements conducted in the time domain, it has been verified that PD frequencies lie between $10 \mathrm{KHz}$ and $15 \mathrm{MHz}$. Time domain data are also captured at various frequencies for 10 power cycles $(200 \mathrm{~ms})$ to verify that we have a PD signal. Typical PD pulses captured at $500 \mathrm{kHz}$ is shown in Figure 5. In Figure 5, distinct PD peaks (encircled ) are evident. The corresponding phase resolved PD pattern is shown in Figure 6. PD clusters are shifted and this is due to the variation in $50 \mathrm{~Hz}$ sync unit used with spectrum analyzer.

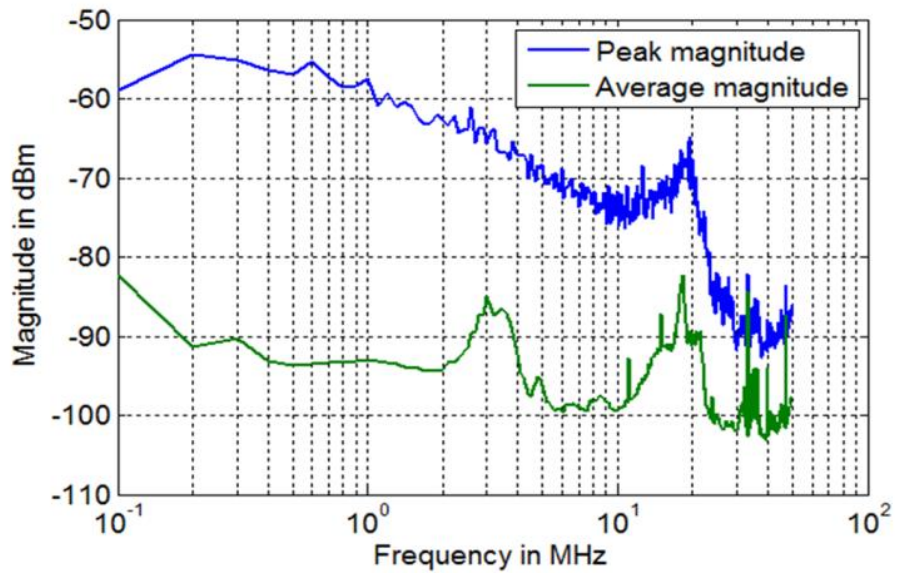

Figure 4 Frequency Spectrum

(Point-Plane, 11.7 kV peak)

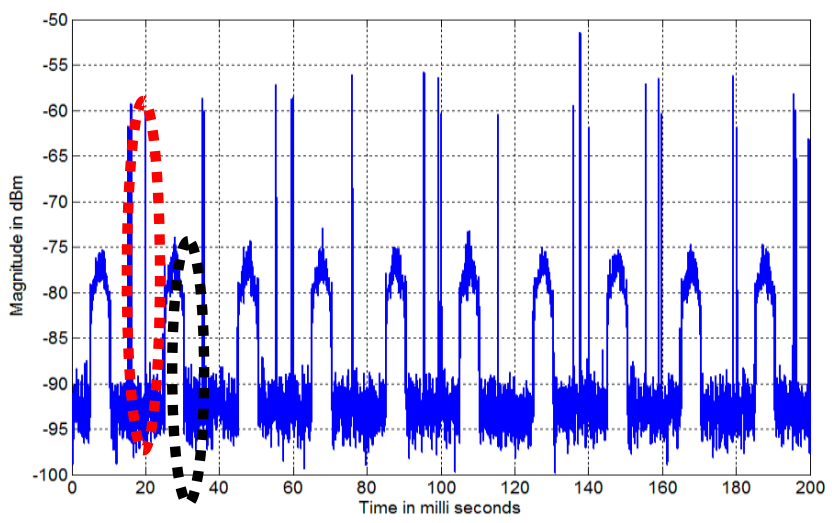

Figure 5 Time Domain PD measurement at $500 \mathrm{KHz}$ (Point-Plane, $11.7 \mathrm{kV}$ peak)

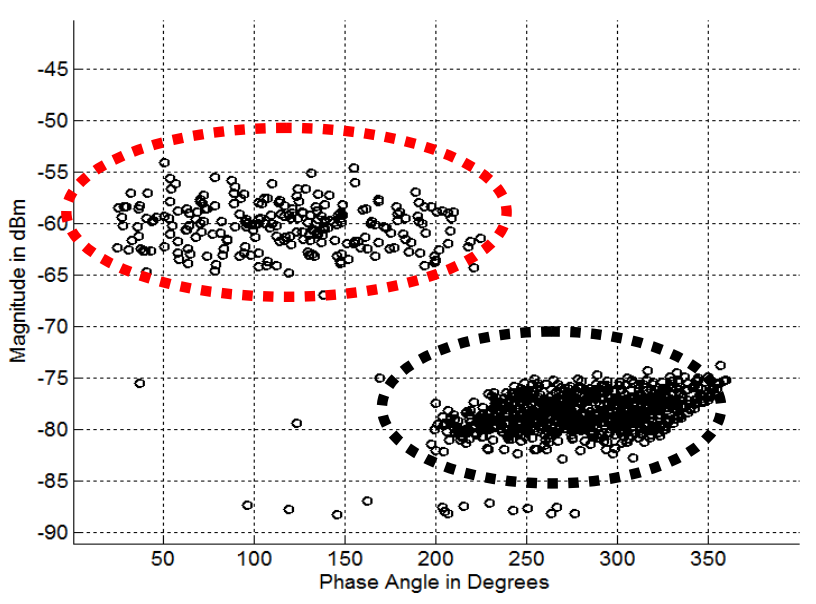

Figure 6 PRPD at $500 \mathrm{KHz}$

(Point-Plane, $11.7 \mathrm{kV}$ peak) 
From the PRPD results, a threshold is applied to extract the PD pulses in the positive half cycle. Average total number of PDs and average PD pulses per 10 power cycles at various frequencies in the measurements conducted at different voltage levels $(-6 \%,-12 \%$ from the initial voltage level) are calculated as shown in Figure 7 and Figure 8.

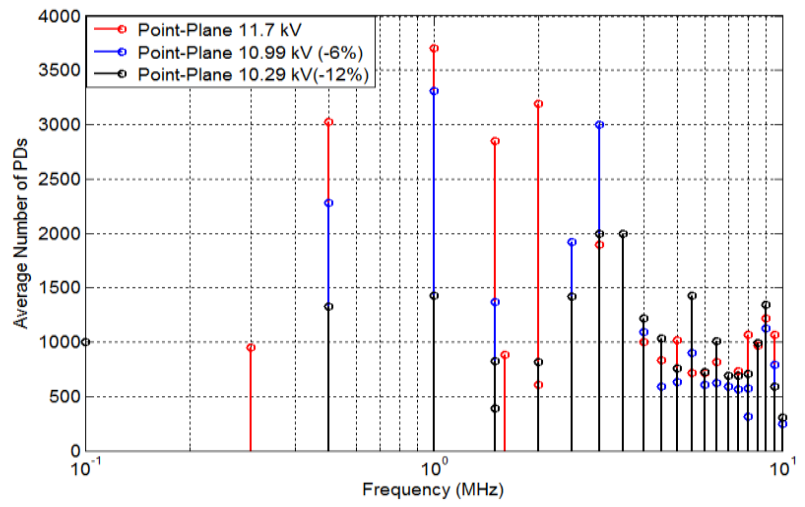

Figure 7 Total number of PDs at various frequencies in $200 \mathrm{mS}$ (Point-Plane, $11.7 \mathrm{kV}$ peak)

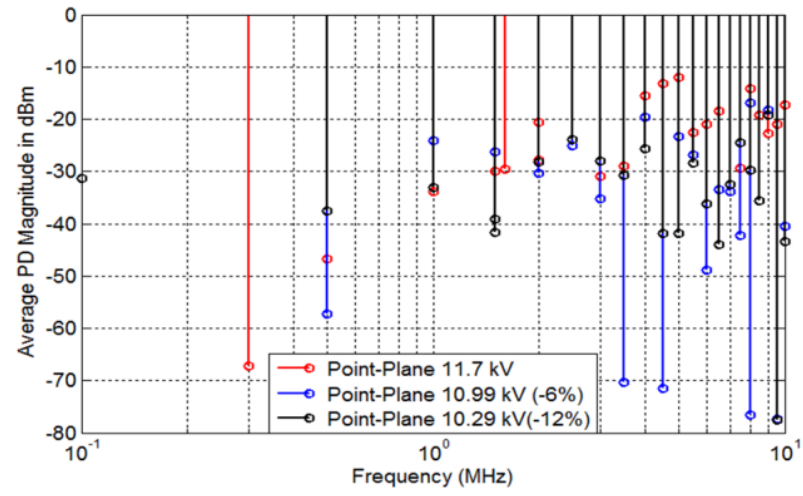

Figure 8 Average PD magnitude at various frequencies in $200 \mathrm{mS}$ (Point-Plane, $11.7 \mathrm{kV}$ peak)

\section{POINT-ROD ELECTRODE}

Same Experiments are repeated for point- rod electrode. PRPD using IEC60270 measurement setup is shown in Figure 9.

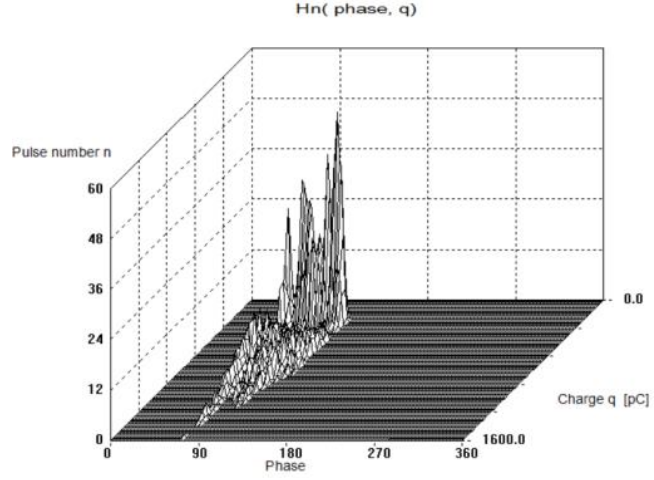

Figure 9 \$-q-n Plot (IEC60270) (Point-Rod, $12.8 \mathrm{kV}$ peak)
Average total number of PDs and average PD pulses per 10 power cycles at various frequencies in the measurements conducted at different voltage levels $(-6 \%,-12 \%$ from the initial voltage level) are calculated as shown in Figure 7 and Figure 8 .

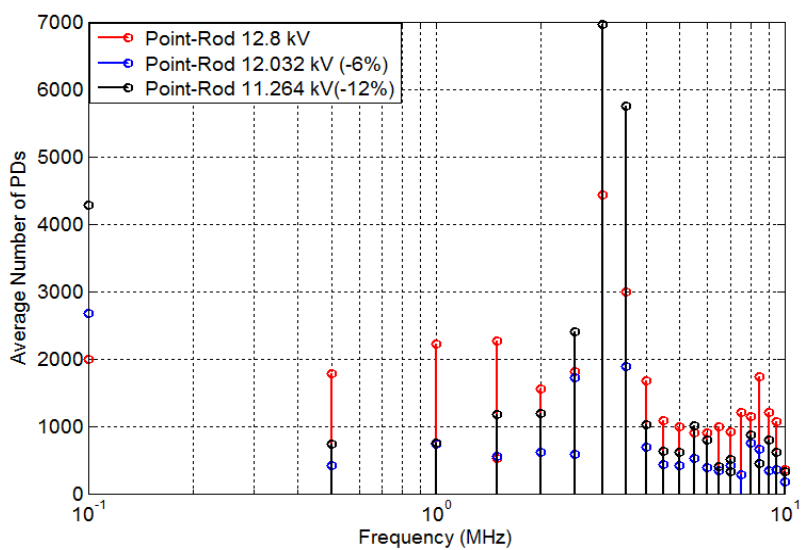

Figure 10 Total number of PDs at various frequencies in $200 \mathrm{mS}$ (Point-Rod, $12.8 \mathrm{kV}$ peak)

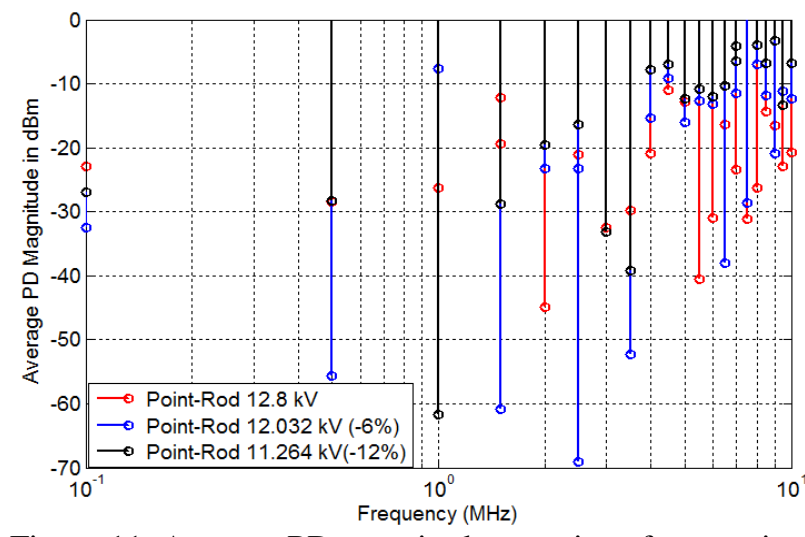

Figure 11. Average PD magnitude at various frequencies in $200 \mathrm{mS}$ (Point-Rod, $12.8 \mathrm{kV}$ peak)

V.POINT-POINT ELECTRODE

Same Experiments are repeated for point- point electrode. PRPD using IEC60270 measurement setup is shown in Figure 12.

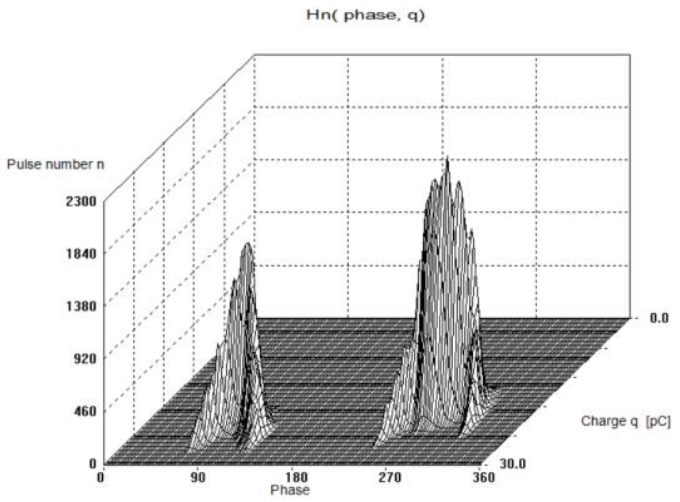

Figure 12 ф-q-n Plot (IEC60270) (Point-Point, 14.5kV) 


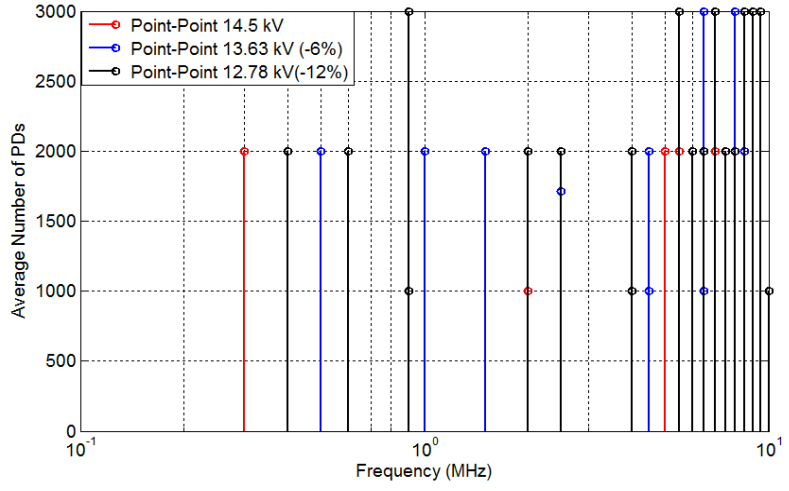

Figure 13. Total number of PDs at various frequencies in $200 \mathrm{mS}$ (Point-Point, $14.5 \mathrm{kV}$ peak)

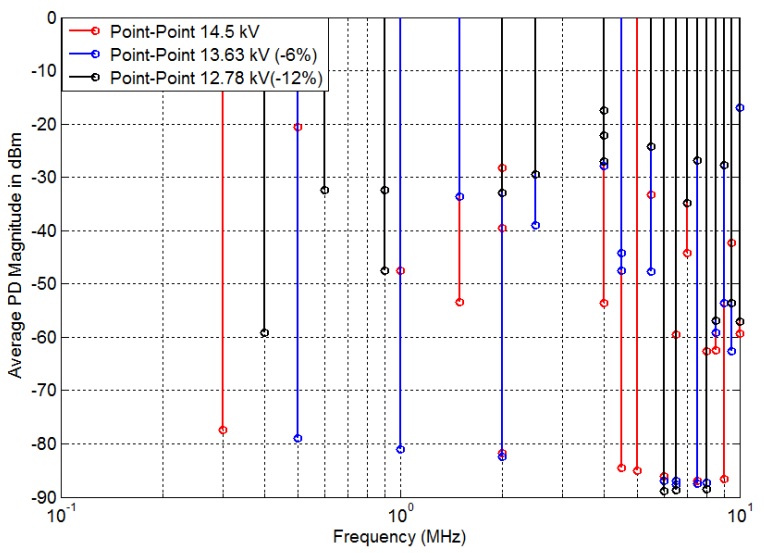

Figure 14 Total number of PDs at various frequencies in $200 \mathrm{mS}$ (Point-Point, $14.5 \mathrm{kV}$ peak)

\section{DISCUSSION AND FURTHER WORK}

Voltage dependence of PD activity can be applied to minimize PD magnitude in high voltage equipment. Currently, industry voltage statutory requirements permit $\pm 6 \%$ tolerance on nominal voltage for distribution networks. Such $\pm 6 \%$ voltage variation on PD initiation was investigated in the laboratory. Three different types of defects causing PD in cables such as point-point, point-plane and point-rod were considered. PD measurements were conducted at nominal, $-6 \%$ and $-12 \%$ from nominal voltage were made. PD was measured using IEC60270 measurement setup along with frequency domain measurement using spectrum analyzer. It has been observed that the total number of PDs and average PD magnitude varies at each frequency within the wide-band PD frequency range. This variation is also dependent on the type of electrode geometry which is an approximation to the PD sites in cables. This shows that voltage reduction does reduce PD activity in cables and they also have an effect on the frequency components of PD. Since high frequency components are attenuated during propagation in cables, to quantify the effect of voltage reduction on PD, frequency domain measurement of PD could be considered.
Further work includes extending the frequency domain approach for measuring PD in power cables.

\section{ACKNOWLEDGEMENT}

The authors wish to express sincere gratitude for funding support from EPSRC Hubnet, the Supergen Energy Networks Hub, under Grant EP/N030028/1.

\section{REFERENCES}

1. Faisal Peer Mohamed, W.H.Siew., John J. Soraghan, Scott Munro Strachan,Jamie McWilliam, Remote monitoring of partial discharge data from insulated power cables. IET Science, Measurement and Technology, 2014.

2. Faisal Peer Mohamed, W.H.Siew., John J. Soraghan, Scott Munro Strachan,Jamie McWilliam ., The use of power frequency current transformers as partial discharge sensors for underground cables. IEEE Transactions on Dielectrics and Electrical Insulation, 2013. 20(3): p. 814 - 824.

3. IEC 60270, I.I.S., High Voltage Test Techniques Partial Discharge Measurements, in International Electrotechnical Commission (IEC), Geneva, Switzerland, 3 rd edition, 2000. 2000: Geneva, Switzerland.

4. Pattanadech, N., et al. The possibility of using a needle plane electrode for partial discharge inception voltage measurement. in 2013 Annual Report Conference on Electrical Insulation and Dielectric Phenomena. 2013. 